\title{
On the critical behaviour of random anisotropy magnets
}

\author{
M.Dudka ${ }^{1}$, R.Folk ${ }^{2}$, Yu.Holovatch ${ }^{1,3}$ \\ 1 Institute for Condensed Matter Physics \\ of the National Academy of Sciences of Ukraine, \\ 1 Svientsitskii Str., 79011 Lviv, Ukraine \\ 2 Institut für Theoretische Physik, Johannes Kepler Universität Linz, \\ A-4040 Linz, Austria \\ 3 Ivan Franko National University of Lviv, \\ 12 Drahomanov Str., 79005 Lviv, Ukraine
}

Received August 14, 2000

The effect of a local anisotropy of random orientation on a ferromagnetic phase transition is studied. To this end, a model of a random anisotropy magnet is analysed by means of a field theoretical renormalization group approach. The one-loop result of Aharony about the absence of a 2 nd order phase transition for isotropic distribution of random anisotropy axis at space dimension $d<4$ is corroborated.

Key words: random anisotropy, renormalization group, critical exponents

PACS: $61.43 .-j, 64.60 . A k$

\section{Introduction}

Even a weak structural disorder may have a crucial effect on the critical behaviour of different materials, in particular magnets. It can alter not only non-universal thermodynamic characteristics of a magnet but lead to a change of the universality class or modify the low-temperature phase behaviour leading, for instance, to a spinglass phase. Here, one should discriminate between random site, random-field and random anisotropy magnets. A weak quenched disorder preserves 2nd order phase transition in three dimensional $(d=3)$ random site magnets [1] but can destroy this transition in random field systems [2] for $d<4$. Situation is not so clear for the random-anisotropy magnets.

Typical examples of random-anisotropy magnets are amorphous rare-earth transition metal alloys [3]. Some of these systems order magnetically and to describe this ordering it has been proposed [4] to consider a regular lattice of magnetic ions, each of them being a subject to a local anisotropy field of random orientation. The 
Hamiltonian of a random anisotropy model (RAM) reads [4]:

$$
\mathcal{H}=-\sum_{\mathbf{R}, \mathbf{R}^{\prime}} J_{\mathbf{R}, \mathbf{R}^{\prime}} \vec{S}_{\mathbf{R}} \vec{S}_{\mathbf{R}^{\prime}}-D_{0} \sum_{\mathbf{R}}\left(\hat{x}_{\mathbf{R}} \vec{S}_{\mathbf{R}}\right)^{2}
$$

where $\vec{S}_{\mathbf{R}}$ is an $m$-component vector on a lattice site $\mathbf{R}, J_{\mathbf{R}, \mathbf{R}^{\prime}}$ is an exchange interaction, $D_{0}$ is an anisotropy strength, and $x_{\mathbf{R}}$ is an unit vector pointing in the local (quenched) random direction of an uniaxial anisotropy. Note that randomness is present in the Hamiltonian (1) only for $m>1$ : at $m=1$ the second term equals constant and leads to a shift in free energy of the resulting regular (Ising) model.

The model was investigated by a variety of techniques including mean-field theory [5], computer simulations [6], $1 / m$-expansion [7], renormalization group $\varepsilon$ expansion [8-10]. Limiting case of an infinite anisotropy was a subject of a detailed study as well $[12,13]$. However the question concerning the nature of lowtemperature phase in RAM is not completely clear up to now. Among the possible low-temperature phases there are discussed ferromagnetic ordering $[5,6]$, spin-glass phase $[6,7]$, quasi long-range ordering [14].

Note that the nature of ordering is connected with the distribution of random variables $\hat{x}_{\mathbf{R}}$ in (1). For the isotropic distribution, arguments similar to those applied by Imry and Ma [15] for a random-field Ising model bring about the absence of the ferromagnetic order for space dimensions $d<4[10,11]$, whereas anisotropic distributions may lead to a ferromagnetic order [16].

To get a correct description of critical behaviour it is standard now to rely on a renormalization group ( $\mathrm{RG}$ ) results. Application of Wilson RG technique to RAM with the isotropic distribution of a local anisotropy axis lead Aharony [8] to conjecture about possible "runaway" solutions of recursion equations. Such a behaviour was interpreted as a smeared transition. However this result was obtained in the first order of $\varepsilon$-expansion and remains to be confirmed within a more refined analysis. Here, we apply a field theoretical RG technique and study RG equations in two loop approximation in order to check the conjecture of Aharony [8]. The paper is organized as follows: in section 2 we describe the model and obtain the RG functions within massive field theory scheme. Fixed points and their stability are analysed in section 3 by means of an $\varepsilon$-expansion to order $\varepsilon^{2}$ and by resummation of a $d=3$ series. Section 4 summarizes our results.

\section{The model and the renormalization}

For a given configuration of quenched random variables $\hat{x}_{\mathbf{R}}$ in (1), the partition function of RAM may be written in the form of functional integral of a Gibbs distribution with the effective Hamiltonian:

$$
\mathcal{H}\left(\hat{x}_{\mathbf{R}}, \vec{\phi}\right)=-\int \mathrm{d}^{d} R\left\{\frac{1}{2}\left[r_{0}|\vec{\phi}|^{2}+|\vec{\nabla} \vec{\phi}|^{2}\right]-D_{1}\left(\hat{x}_{\mathbf{R}} \vec{\phi}\right)^{2}+v_{0}|\vec{\phi}|^{4}+\ldots\right\},
$$

where $D_{1}$ is proportional to $D_{0}, r_{0}$ and $v_{0}$ are defined by $D_{0}$ and familiar bare couplings of an $m$-vector model, and $\vec{\phi} \equiv \vec{\phi}_{\mathbf{R}}$ is a $m$-dimensional vector. The case 
of isotropic distribution of a local anisotropy axis we consider here corresponds to the situation when the random vector $\hat{x}_{\mathbf{R}}$ points with equal probability in any direction of $m$-dimensional hyperspace. In order to deal with quenched averaging one introduces $n$ replicas of the Hamiltonian (2) and ends with the following effective Hamiltonian [8]:

$$
\mathcal{H}_{\text {eff }}=-\int \mathrm{d}^{d} R\left\{\frac{1}{2}\left[\mu_{0}{ }^{2}|\vec{\varphi}|^{2}+|\vec{\nabla} \vec{\varphi}|^{2}\right]+u_{0}|\vec{\varphi}|^{4}+v_{0} \sum_{\alpha=1}^{n}\left|\vec{\phi}^{\alpha}\right|^{4}+w_{0} \sum_{\alpha, \beta=1}^{n} \sum_{i, j=1}^{m} \phi_{i}^{\alpha} \phi_{j}^{\alpha} \phi_{i}^{\beta} \phi_{j}^{\beta}\right\},
$$

where $\mu_{0}$ is bare mass and bare couplings $u_{0}>0, v_{0}>0, w_{0}<0$. Furthermore, $u_{0}, w_{0}$ are related to appropriate cumulants of the random vector $\hat{x}_{\mathbf{R}}$ distribution function and their ratio equals $w_{0} / u_{0}=-m$. Note that the symmetry of $u_{0}$ and $v_{0}$ terms corresponds to the random site $m$-vector model [17]. However the $u_{0}$-term has an opposite sign.

In order to study long-distance properties of the Hamiltonian (3), we use the field-theoretical RG approach. We apply the massive field theory renormalization scheme [18] performing renormalization at fixed space dimension $d$ and zero external momenta. For the $\beta$-functions in two-loop approximation in replica limit $n=0$ we get:

$$
\begin{aligned}
\beta_{u}= & -\varepsilon\left\{u-\frac{1}{6}\left[8 u^{2}+2(m+2) u v+2 v w+2(m+1) u w+3 w^{2}\right]+\frac{1}{9}\left[44 u^{3}\right.\right. \\
& +24(m+2) v u^{2}+2(3 m+6) u v^{2}+2(6 m+24) u v w+2(12 m+12) w u^{2} \\
& \left.+(3 m+45) u w^{2}+2(m+8) v w^{2}+4 v^{2} w+(3 m+9) w^{3}\right] i_{1}+\frac{2}{9}\left[2 u^{3}\right. \\
& \left.\left.+2(m+2) v u^{2}+(m+2) u v^{2} \frac{m+3}{2} w^{2} u+2(m+2) u v w+2(m+1) u^{2} w\right] i_{2}\right\}, \\
\beta_{v}= & -\varepsilon v\left\{1-\frac{1}{6}[(m+8) v+12 u+2(m+5) w]+\frac{1}{9}\left[2(5 m+22) v^{2}+24(m+5) u v\right.\right. \\
& \left.+84 u^{2}+2(14 m+58) w v+4(9 m+33) u w+(17 m+67) w^{2}\right] i_{1} \\
& \left.+\frac{2}{9}\left[2 u^{2}+2(m+2) u v+(m+2) v^{2}+\frac{m+3}{2} w^{2}+2(m+2) v w+2(m+1) u w\right] i_{2}\right\}, \\
\beta_{w}= & -\varepsilon w\left\{1-\frac{1}{6}[(m+4) w+12 u+4 v]+\frac{1}{9}\left[(5 m+27) w^{2}+4(6 m+15) u w\right.\right. \\
& \left.+2(5 m+22) v w+4(3 m+18) u v+84 u^{2}+2(m+6) v^{2}\right] i_{1} \\
& \left.+\frac{2}{9}\left[2 u^{2}+2(m+2) u v+(m+2) v^{2}+\frac{m+3}{2} w^{2}+2(m+2) v w+2(m+1) u w\right] i_{2}\right\} .
\end{aligned}
$$

Here, $u, v, w$ are renormalized couplings and $i_{1}, i_{2}$ are two-loop integrals [19]. For the space dimension $d=3$ they equal $i_{1}=1 / 6, i_{2}=-2 / 27$ [20]. Zeroes of the $\beta$-functions determine the coordinates of fixed points (FPs). The stable FP is defined as the FP where the stability matrix $B_{i j}=\partial \beta_{u_{i}} / \partial u_{j}, u_{i}=\{u, v, w\}$ possess eigenvalues with positive real parts. 
Table 1. Fixed points in $\varepsilon$-expansion. Here, $x_{ \pm}=\left(m-2 \pm \sqrt{(m-2)^{2}+48}\right) / 8$, $y_{ \pm}=\left(m-2-2 m z \pm \sqrt{(m-2-2 m z)^{2}+4(12-8 z)}\right) / 8, z=(m+6) /(m+8)$.

The second order contributions $u_{i}, v_{i}, w_{i}$ are given in the appendix.

\begin{tabular}{|l|c|c|c|}
\hline & $u^{*}$ & $v^{*}$ & $w^{*}$ \\
\hline I. & 0 & 0 & 0 \\
II. & 0 & $\frac{6}{m+8} \varepsilon+18 \frac{3 m+14}{(m+8)^{3}} \varepsilon^{2}$ & 0 \\
III. & $\frac{6}{8} \varepsilon+\frac{63}{128} \varepsilon^{2}$ & 0 & 0 \\
IV. & $-\frac{3(4-m)}{8(m-1)} \varepsilon+u_{\mathrm{IV}} \varepsilon^{2}$ & $\frac{3}{2(m-1)} \varepsilon+v_{\mathrm{IV}} \varepsilon^{2}$ & $\frac{6}{12 x_{+}+m+4} \varepsilon+w_{\mathrm{V}} \varepsilon^{2}$ \\
V. & $\frac{6 x_{+}}{12 x_{+}+m+4} \varepsilon+u_{\mathrm{V}} \varepsilon^{2}$ & 0 & $\frac{6}{12 x_{-}+m+4} \varepsilon+w_{\mathrm{VI}} \varepsilon^{2}$ \\
VI. & $\frac{6 x_{-}}{12 x_{-}+m+4} \varepsilon+u_{\mathrm{VI}} \varepsilon^{2}$ & 0 & $6 z$ \\
VII. & $\frac{6 y_{+}}{12 y_{+}-4 z+m+4} \varepsilon+u_{\mathrm{VII}} \varepsilon^{2}$ & $-\frac{6 z}{12 y_{+}-4 z+m+4} \varepsilon+v_{\mathrm{VII}} \varepsilon^{2}$ & $\frac{6 z}{12 y_{+}-4 z+m+4} \varepsilon+w_{\mathrm{VII}} \varepsilon^{2}$ \\
VIII. & $\frac{6}{12 y_{-}-4 z+m+4} \varepsilon+u_{\mathrm{VIII}} \varepsilon^{2}$ & $-\frac{6}{12 y_{-}-4 z+m+4} \varepsilon+v_{\mathrm{VIII}} \varepsilon^{2}$ & $\frac{6}{12 y_{-}-4 z+m+4} \varepsilon+w_{\mathrm{VIII}} \varepsilon^{2}$ \\
\hline
\end{tabular}

\section{Fixed points and their stability}

$\varepsilon$-expansion. As it was mentioned in the introduction the only known RG results for RAM with isotropic distribution of the local anisotropy axis are those obtained in the first order in $\varepsilon$ [8]. They can be reproduced from formulas (4)-(6) putting twoloop contributions equal to zero. In particular, we get eight FPs with coordinates given in table 1 (in order to recover the results of [8] we extract the value of one-loop integral $\sim 1 / \varepsilon$ from conventionally normalized couplings: see note [19]). In the first order of $\varepsilon$-expansion all FPs with $u>0, v>0, w<0$ appear to be unstable for $\varepsilon>0$ except for the "polymer" $O(n=0)$ FP III which is stable for all $m$. However, the presence of a stable FP is not a sufficient condition for the 2nd order phase transition. The FP should be accessible from the initial values of couplings and it is not the case for the location of FPs shown in figure 1. Indeed starting from the region of initial conditions (denoted by cross in the figure) for zero value of $v$ one meets a separatrix joining unstable FPs I and VI and will never reach the stable FP III. As far as both FPs I and VI are strongly unstable with respect to $v$, FP III is not accessible for arbitrary positive $v$ either. Finally, one ends up with the conclusion about the absence of the 2nd order phase transition in the model as runaway solutions of the RG equations show.

Second-order contributions in $\varepsilon$ to the FP coordinates are displayed in table 1 as well. The main question of interest here is whether the above described picture of the runaway solution is not an artifact of an $\varepsilon$-expansion. To shed light on this issue we will hereinafter use a more refined analysis of FPs and their stability.

$\boldsymbol{d}=3$ series. Another way of analysing the series for the RG functions (4)-(6) is to consider them directly for the dimension of interest $d=3$ [18]. As it is known, the series of this type are asymptotic at best and a resummation procedure is to be applied in order to obtain reliable data on their basis. Here, we will make use of Padé-Borel resummation techniques [21] first writing the RG functions as resolvent 


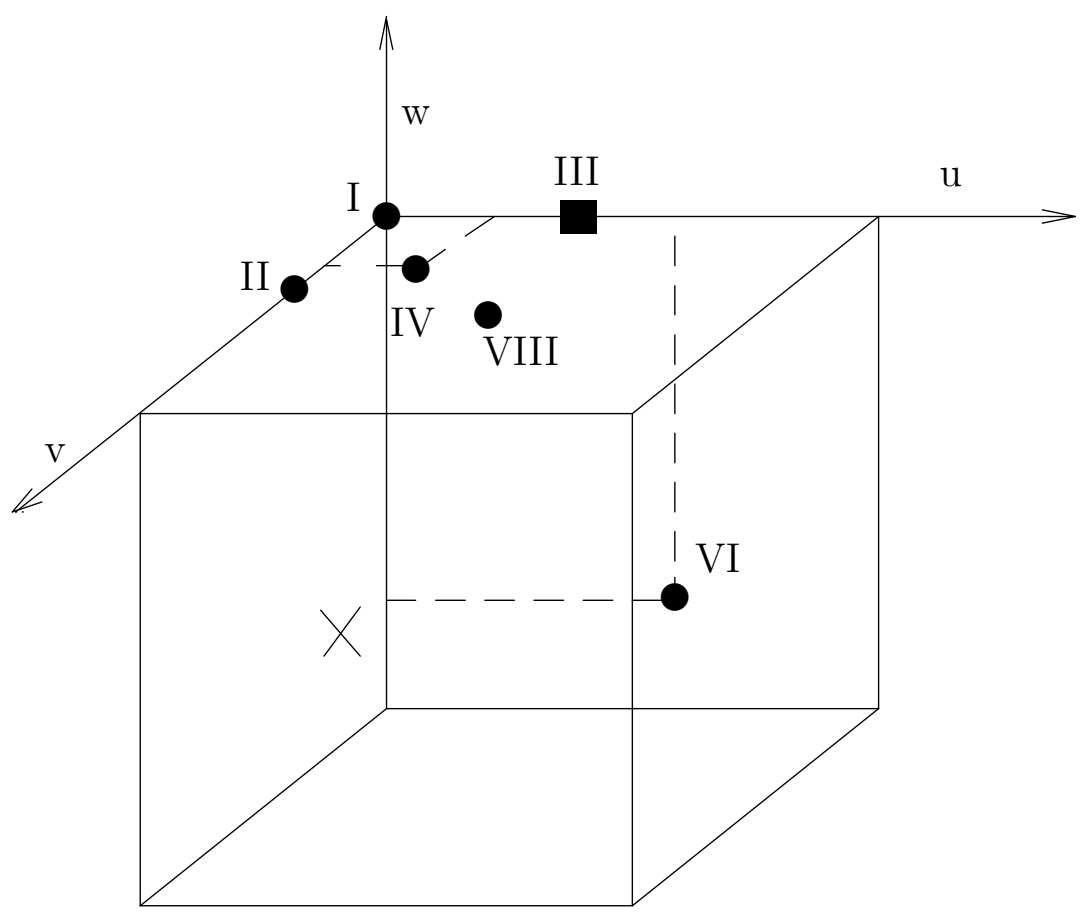

Figure 1. Fixed points of the RAM with isotropic distribution of a local anisotropy axis. The only fixed points located in the octant $u>0, v>0, w<0$ are shown. Filled box shows the stable fixed point, cross denotes typical initial values of couplings.

Table 2. Resummed values of the fixed points in two-loop approximation for $d=3$.

\begin{tabular}{|c|c|c|c|c|}
\hline & $m$ & $u^{*}$ & $v^{*}$ & $w^{*}$ \\
\hline \hline I & $\forall m$ & 0 & 0 & 0 \\
\hline II & 2 & 0 & 0.9107 & 0 \\
& 3 & 0 & 0.8102 & 0 \\
& 4 & 0 & 0.7275 & 0 \\
\hline III & $\forall m$ & 1.1857 & 0 & 0 \\
\hline IV & 2 & -0.0322 & 0.9454 & 0 \\
& 3 & 0.1733 & 0.6460 & 0 \\
& 4 & 0.2867 & 0.4851 & 0 \\
\hline VI & 2 & 1.4650 & 0 & -1.6278 \\
\hline VIII & 2 & 0.7517 & 0.7072 & -0.3984 \\
& 3 & 0.8031 & 0.5463 & -0.3305 \\
& 4 & 0.8349 & 0.4545 & -0.2888 \\
\hline
\end{tabular}

series [22] in one auxiliary variable and then performing resummation. Numerical values of the FPs are given in table 2. Resummed two-loop results qualitatively confirm the picture obtained in the first order in $\varepsilon$-expansion: stability of the FPs does not change after resummation. This supports a conjecture of Aharony [8] about the absence of accessible stable FP for the RAM with isotropic distribution of the local anisotropy axis.

However the applied procedure of resummation fails to give a correct description of $\beta$-functions for large negative $w$. In particular, in the region of couplings in the vicinity of FP VI we get real values of the $\beta$-functions and solution for $m=2$ only. This is caused by the appearance of poles in integral rep- 
resentations of the resummed $\beta$-functions. To deal with the poles one can take the principal values of corresponding integrals but we will exclude such cases from our analysis. Studying the evolution of FPs upon application of the resummation procedure we have found one more $\mathrm{FP}$, which is not present in the $\varepsilon$-expansion analysis. This one we consider as an artifact of the resummation procedure and do not display it in table 2. In the other FPs we recover the two-loop results for $O(m)$ (FP II), polymer $O(n=0)$ (FP III) and diluted $m$-vector (FP IV) models. FP VIII contains all three couplings but is both unstable and non-accessible from the initial values of couplings.

\section{Conclusions}

In this paper we applied a field theoretical RG approach to analyse the critical behaviour of RAM with isotropic distribution of a local anisotropy axis. The origin of a low temperature phase in this model is not completely clear. General arguments based on an estimate of the energy for the formation of magnetic domains [15] lead to a conclusion about the absence of ferromagnetic order for $d<4[10,11]$. However, these arguments do not take into account the entropy which may be important for disordered systems [13].

In the RG analysis, the absence of a ferromagnetic second order phase transition corresponds to a lack of stable FP of the RG transformation. However, in the case of RAM with isotropic distribution of a local anisotropy axis the scenario differs. Our two-loop calculation bring about the presence of a $O(n=0)$ symmetric FP which is stable for any value of $m$. However, this FP is not accessible for the initial values of couplings. We checked the location of the FP up to the second order in $\varepsilon$-expansion and by means of a fixed $d=3$ technique refined by Padé-Borel resummation. Our analysis supports the conjecture of Aharony based on linear in $\varepsilon$ results concerning runaway solutions of $\mathrm{RG}$ equations for the RAM with isotropic distribution of a local anisotropy axis.

It is worth mentioning here that anisotropic distribution of a local anisotropy axis may lead to ferromagnetism by a second order phase transition scenario [16]. The analysis of effective and asymptotic critical behaviour in this case will be a subject of a separate study.

Yu.H. acknowledges helpful discussions with Mykola Shpot. This work has been supported in part by "Österreichische Nationalbank Jubiläumsfonds" through the grant No 7694 .

\section{Appendix}

In this appendix, we perform the $\varepsilon$-expansion for the RAM with isotropic distribution of a local anisotropy axis. A procedure allowing to obtain the $\varepsilon$-expansion from the RG functions written in massive scheme for a fixed $d$ is well known. To this end, one should substitute loop integrals by their expansions in $\varepsilon$ and proceed in a common way (see e.g. [23]). In particular, substituting integrals $i_{1}, i_{2}$ in (4)-(6) by 
Table 3. Numerical values of coefficients at the contributions $\sim \varepsilon^{2}$ to the fixed points coordinates for some $m$.

\begin{tabular}{|c|c|c|c|c|c|c|}
\hline$m$ & $u_{\mathrm{IV}}$ & $u_{\mathrm{V}}$ & $u_{\mathrm{VI}}$ & $u_{\mathrm{VII}}$ & $u_{\mathrm{VIII}}$ & $v_{\mathrm{IV}}$ \\
\hline 2 & -3.8906 & 0.2517 & 0.3525 & -0.0242 & -0.6352 & 3.2578 \\
3 & -0.6665 & 0.2581 & -8.6484 & -0.4935 & -0.5566 & 0.8346 \\
4 & -0.2292 & 0.2654 & 27165.9534 & -1.0550 & -0.5558 & 0.5 \\
\hline & $v_{\mathrm{VII}}$ & $v_{\mathrm{VIII}}$ & $w_{\mathrm{V}}$ & $w_{\mathrm{VI}}$ & $w_{\mathrm{VII}}$ & $w_{\mathrm{VIII}}$ \\
\hline 2 & -6.4311 & 0.8791 & 0.1889 & -0.3313 & 4.7905 & 1.2583 \\
3 & -7.8255 & 0.2024 & 0.1441 & 12.3348 & 5.6388 & 1.2962 \\
4 & -9.6584 & -0.1118 & 0.1105 & -39381.2980 & 6.6343 & 1.2650 \\
\hline
\end{tabular}

their expansions $i_{1} \simeq 1 / 2+\varepsilon / 4+\ldots, i_{2} \simeq-\varepsilon / 8+\ldots$ [24] we get the expressions for the fixed point coordinates given in table 1 with the accuracy $\varepsilon^{2}$. We do not display there the second order contributions $u_{i}, v_{i}, w_{i}$ as functions of $m$, because they are too cumbersome. Instead in table 3 we list their numerical values for $m=2,3,4$.

\section{References}

1. A weak quenched random site dilution is irrelevant if the heat capacity critical exponent $\alpha_{\text {pure }}$ of the undiluted system is negative (Harris A.B. // J. Phys. C, 1974, vol. 7, p. 1671). For positive $\alpha_{\text {pure }}$ dilution causes change in the universality class (see e.g. Folk R., Holovatch Yu., Yavors'kii T. // Phys. Rev. B, 2000, vol. 61, p. 15114 for a recent review).

2. See e.g. review of Belanger D.P., Young A.P. // JMMM, 1991, vol. 100, p. 272.

3. Cochrane R.W., Harris R., Zuckermann M.J. // Phys. Rep., 1978, vol. 1, p. 1.

4. Harris R., Plischke M., Zuckermann M.J. // Phys. Rev. Lett., 1973, vol. 31, p. 160.

5. Derrida B., Vannimenus J. // J. Phys. C, 1980, vol. 13, p. 3261; Harris R., Zobin D. // J. Phys. F, 1977, vol. 7, p. 337; Callen E., Liu Y.J., Cullen J.R. // Phys. Rev. B, 1977, vol. 16, p. 263; Patterson J.D., Gruzalski G.R., Sellmyer D.J. // Phys. Rev. B, 1978, vol. 18, p. 1377; Alben R., Becker J.J., Chi M.C. // J. Appl. Phys., 1978, vol. 49, p. 1653.

6. Chi M.C., Alben R. // J. Appl. Phys., 1977, vol. 48, p. 2987; Chi M.C., Egami T. // J. Appl. Phys., 1979, vol. 50, p. 1651; Harris R., Sung S.H. // J. Phys. F, 1978, vol. 8, p. L299; Jayaprakash C., Kircpatrick S. // Phys. Rev. B, 1980, vol. 21, p. 4072.

7. Goldschmidt Y.Y. // Nucl. Phys. B, 1983, vol. 225, p. 123; Goldschmidt Y.Y. // Phys. Rev. B, 1984, vol. 30, p. 1632; Khurana A., Jagannathan A., Kosterlitz M.J. // Nucl. Phys. B, 1984, vol. 240, p. 1; Jagannathan A., Schaub B., Kosterlitz M.J. // Nucl. Phys. B, 1986, vol. 265, p. 324; Goldschmidt Y.Y., Aharony A. // Phys. Rev. B, 1985, vol. 32, p. 264.

8. Aharony A. // Phys. Rev. B, 1975, vol. 12, p. 1038.

9. Chen J.H., Lubensky T.C. // Phys. Rev. B, 1977, vol. 16, p. 2106.

10. Pelcovits R.A., Pytte E., Rudnick J. // Phys. Rev. Lett., 1978, vol. 40, p. 476; Pelcovits R.A. // Phys. Rev. B, 1979, vol. 19, p. 465; Pytte E. Phys. Rev. B, 1978, vol. 18, p. 5046 . 
11. Ma S.-k., Rudnick J. // Phys. Rev. Lett., 1978, vol. 40, p. 589.

12. Bray A.J., Moore M.A. // J. Phys. C, 1985, vol. 18, p. L139; Harris A.B., Caflisch R.G., Banavar J.R. // Phys. Rev. B, 1987, vol. 35, p. 4929.

13. Fischer K.H., Zippelius A. // J. Phys. C, 1985, vol. 18, p. L1139.

14. Aharony A., Pytte E. // Phys. Rev. Lett., 1980, vol. 19, p. 1583; Fisch R. // Phys. Rev. B, 1998, vol. 57, p. 269; Chakrabati J. // Phys. Rev. Lett., 1998, vol. 81, p. 385; Feldman D.E. // JETP Lett., 1999, vol. 70, p. 135.

15. Imry Y., Ma S.-k. // Phys. Rev. Lett., 1975, vol. 35, p. 1399.

16. Mukamel D., Grinstein G. // Phys. Rev. B, 1982, vol. 25, p. 381; Korzhenevskii A.L., Luzhkov A.A. // Sov. Phys. JETP, 1988, vol. 94, p. 250.

17. Grinstein G., Luther A. // Phys. Rev. B, 1976, vol. 13, p. 1329.

18. Parisi G. 1973 (unpublished); Parisi G. // J. Stat. Phys., 1980, vol. 23, p. 49.

19. We absorb the value of a one-loop integral into normalization of couplings and twoloop integrals.

20. Nickel B.G., Meiron D.I., Baker G.A. Jr. Univ. of Guelph Report, 1977 (unpublished).

21. Baker G.A. Jr., Nickel B.G., Meiron D.I. // Phys. Rev. B, 1978, vol. 17, p. 1365.

22. Watson P.J.S. // J. Phys. A., 1974, vol. 7, p. L167.

23. Amit D.J. Field Theory, the Renormalization Group, and Critical Phenomena. Singapore, World Scientific, 1984.

24. Brezin E., Le Guillou J.C., Zinn-Justin J. // Phys. Rev. D, 1973, vol. 8, p. 434.

\section{Критична поведінка магнетиків із випадковою анізотропією}

$$
\text { М.Дудка }{ }^{1} \text {, Р.Фольк }{ }^{2} \text {, Ю.Головач }{ }^{1,3}
$$

1 Інститут фізики конденсованих систем НАН України, 79011 Львів, вул. Свєнціцького, 1

2 Інститут теоретичної фізики, Університет Йоганна Кеплера в Лінці, А-4040 Лінц, Австрія.

3 Львівський національний університет ім. І. Франка, 79005 Львів, вул. Драгоманова, 12

Отримано 14 серпня 2000 р.

Досліджується вплив локальної анізотропії випадкової орієнтації на феромагнітний фазовий перехід. 3 цією метою за допомогою методу теоретико-польової ренормалізаційної групи проведено аналіз моделі магнетика з випадковою анізотропією. Підтверджено однопетлевий результат Агароні про відсутність фазового переходу другого роду для ізотропного розподілу осі випадкової анізотропії при вимірності простору $d<4$.

Ключові слова: випадкова анізотропія, ренормалізаційна група, критичні показники

PACS: 61.43.-j, 64.60.Ak 